\title{
Correction to: Proposal and evaluation for color constancy CAPTCHA
}

\author{
Shotaro Usuzaki ${ }^{1} \cdot$ Kentaro Aburada $^{1} \cdot$ Hisaaki Yamaba ${ }^{1} \cdot$ Tetsuro Katayama $^{1} \cdot$ Masayuki Mukunoki $^{1} \cdot$ Mirang Park $^{2}$. \\ Naonobu Okazaki ${ }^{1}$
}

Published online: 17 November 2021

(c) International Society of Artificial Life and Robotics (ISAROB) 2021

\section{Correction to:}

Artificial Life and Robotics (2021) 26:291-296

https://doi.org/10.1007/s10015-021-00679-x

In the original publication of the article, on Figs. 4 and 5, "CNN" should read as "MLP".

In addition, under the section "4.2 Human and machine success rate", on the paragraph "For evaluation of the machine success ...", in the following sentence "In the attack experiment, we applied the famous color ..." CNN (Convolutional Neural Network) should be corrected as MLP (Multilayer perceptron).

The correct sentence should read as "In the attack experiment, we applied the famous color constancy algorithm, Gray-World, Max-RGB, Gray-Edge [11], Second Derivative Gray-Edge, and MLP (Multilayer perceptron) to images that were saved in the experiment for the human success rate."

Publisher's Note Springer Nature remains neutral with regard to jurisdictional claims in published maps and institutional affiliations.

These errors were caused by the authors.

The original article can be found online at https://doi.org/10.1007/ s10015-021-00679-x.

Kentaro Aburada

aburada@cs.miyazaki-u.ac.jp

1 University of Miyazaki, 1-1, Gakuen Kibanadai-nishi, Miyazaki, Miyazaki 889-2192, Japan

2 Kanagawa Institute of Technology, 1030, Shimo-Ogino, Atsugi, Kanagawa 243-0292, Japan 